\title{
INITIAL VALUE PROBLEMS AND WEYL-TITCHMARSH THEORY FOR SCHRÖDINGER OPERATORS WITH OPERATOR-VALUED POTENTIALS
}

\author{
Fritz GeSZTESy, RUdi WEIKARD AND MAXIM ZinCHENKO
}

Abstract. We develop Weyl-Titchmarsh theory for self-adjoint Schrödinger operators $H_{\alpha}$ in $L^{2}((a, b) ; d x ; \mathscr{H})$ associated with the operator-valued differential expression $\tau=-\left(d^{2} / d x^{2}\right)+$ $V(\cdot)$, with $V:(a, b) \rightarrow \mathscr{B}(\mathscr{H})$, and $\mathscr{H}$ a complex, separable Hilbert space. We assume regularity of the left endpoint $a$ and the limit point case at the right endpoint $b$. In addition, the bounded self-adjoint operator $\alpha=\alpha^{*} \in \mathscr{B}(\mathscr{H})$ is used to parametrize the self-adjoint boundary condition at the left endpoint $a$ of the type

$$
\sin (\alpha) u^{\prime}(a)+\cos (\alpha) u(a)=0,
$$

with $u$ lying in the domain of the underlying maximal operator $H_{\max }$ in $L^{2}((a, b) ; d x ; \mathscr{H})$ associated with $\tau$. More precisely, we establish the existence of the Weyl-Titchmarsh solution of $H_{\alpha}$, the corresponding Weyl-Titchmarsh $m$-function $m_{\alpha}$ and its Herglotz property, and determine the structure of the Green's function of $H_{\alpha}$.

Developing Weyl-Titchmarsh theory requires control over certain (operator-valued) solutions of appropriate initial value problems. Thus, we consider existence and uniqueness of solutions of 2nd-order differential equations with the operator coefficient $V$,

$$
\left\{\begin{array}{l}
-y^{\prime \prime}+(V-z) y=f \text { on }(a, b), \\
y\left(x_{0}\right)=h_{0}, y^{\prime}\left(x_{0}\right)=h_{1},
\end{array}\right.
$$

under the following general assumptions: $(a, b) \subseteq \mathbb{R}$ is a finite or infinite interval, $x_{0} \in(a, b)$, $z \in \mathbb{C}, V:(a, b) \rightarrow \mathscr{B}(\mathscr{H})$ is a weakly measurable operator-valued function with $\|V(\cdot)\|_{\mathscr{B}(\mathscr{H})} \in$ $L_{\mathrm{loc}}^{1}((a, b) ; d x)$, and $f \in L_{\mathrm{loc}}^{1}((a, b) ; d x ; \mathscr{H})$. We also study the analog of this initial value problem with $y$ and $f$ replaced by operator-valued functions $Y, F \in \mathscr{B}(\mathscr{H})$.

Our hypotheses on the local behavior of $V$ appear to be the most general ones to date.

Mathematics subject classification (2010): Primary: 34A12, 34B20, 34B24; Secondary: 47E05. Keywords and phrases: Weyl-Titchmarsh theory, ODEs with operator coefficients, Schrödinger operators.

\section{REFERENCES}

[1] V. M. AdAmjan AND H. LANGer, Spectral properties of a class of rational operator valued functions, J. Operator Th. 33 (1995), 259-277.

[2] S. Agmon, J. CruZ-S Ampedro, ANd I. Herbst, Generalized Fourier transform for Schrödinger operators with potentials of order zero, J. Funct. Anal. 167 (1999), 345-369.

[3] Z. S. Agranovich And V. A. Marchenko, The Inverse Problem of Scattering Theory, Gordon and Breach, New York, 1963.

[4] S. Albeverio, J. F. Brasche, M. M. Malamud, and H. Neidhardt, Inverse spectral theory for symmetric operators with several gaps: scalar-type Weyl functions, J. Funct. Anal. 228 (2005), $144-188$.

[5] B. A. Aliev, Asymptotic behavior of the eigenvalues of a boundary-value problem for a secondorder elliptic operator-differential equation, Ukrain. Math. J. 58 (2006), 1298-1306. 
[6] A. R. ALIEV, On the generalized solution of the boundary-value problem for the operatordifferential equations of the second order with variable coefficients, J. Math. Phys. Anal. Geom. 2 (2006), 87-93.

[7] B. A. ALIEV, Solvability of the boundary-value problem for the second-order elliptic differentialoperator equation with spectral parameter in the equation and boundary conditions, Ukrain. Math. J. 62 (2010), 1-14.

[8] A. R. Aliev And S. S. Mirzoev, On boundary value problem solvability theory for a class of high-order operator-differential equations, Funct. Anal. Appl. 44 (2010), 209-211.

[9] G. D. Allen AND F. J. NARCOWICH, On the representation and approximation of a class of operator-valued analytic functions, Bull. Amer. Math. Soc. 81 (1975), 410-412.

[10] G. D. Allen and F. J. NARCowich, R-operators I. Representation Theory and Applications, Indiana Univ. Math. J. 25 (1976), 945-963.

[11] M. S. Almamedov, M. BaǏramogly, And V. I. Katanova, Trace formulas for a differential equation of even order with unbounded operator coefficient, Sov. Math. Dokl. 43 (1991), 406-410.

[12] M. S. Almamedov, M. BaǏramogly, And V. I. Katanova, On regularized traces of even-order differential equations with unbounded operator coefficients, Diff. Eq. 29 (1993), 1-9.

[13] M. S. Almamedov AND V. I. KatANOVA, A regularized trace of a high-order differential operator with a bounded operational coefficient, Diff. Eqs. 28 (1992), 1-15.

[14] H. Amann, Linear and Quasilinear Parabolic Problems, Monographs in Mathematics, Vol. 89, Birkhäuser, Basel, 1995.

[15] W. Arendt, C. K. Batty, M. Hieber, F. Neubrander, Vector-Valued Laplace Transforms and Cauchy Transforms, Monographs in Mathematics, Vol. 96, Birkhäuser, Basel, 2001.

[16] Yu. Arlinskit, S. Belyi, And E. TseKanovskiI, Conservative Realizations of HerglotzNevanlinna Functions, Operator Theory advances and Applications, Vol. 217, Birkhäuser, Springer, Basel, 2011.

[17] N. Aronszajn And W. F. Donoghue, On exponential representations of analytic functions in the upper half-plane with positive imaginary part, J. Analyse Math. 5 (1956-57), 321-388.

[18] K. As ANo, Notes on Hilbert transforms of vector valued functions in the complex plane and their boundary values, Proc. Japan Acad. 43 (1967), 572-577.

[19] N. M. Aslanova, A trace formula of a boundary value problem for the operator Sturm-Liouville equation, Sib. Math. J. 49 (2008), 959-967.

[20] M. Bairamogly And N. M. Aslanova, Distribution of eigenvalues and trace formula for the Sturm-Liouville operator equation, Ukrain. Math. J. 62 (2010), 1005-1017.

[21] H. BaumgäRtel And M. Wollenberg, Mathematical Scattering Theory, Operator Theory: Advances and Applications, Vol. 9, Birkhäuser, Boston, 1983.

[22] S. V. Belyi AND E. R. TSEKAnOvSKII, Classes of operator $R$-functions and their realization by conservative systems, Sov. Math. Dokl. 44 (1992), 692-696.

[23] R. Benguria And M. Loss, A simple proof of a theorem of Laptev and Weidl, Math. Res. Lett. 7 (2000), 195-203.

[24] C. Bennewitz, Spectral theory in Hilbert space, Lecture Notes, 2008.

[25] Ju. BerezanskiĬ, Expansions in Eigenfunctions of Selfadjoint Operators, Transl. Math. Mongraphs, Vol. 17, Amer. Math. Soc., Providence, R.I., 1968.

[26] M. Š. BIRMAN, S. B. ÈnTINA, The stationary method in the abstract theory of scattering theory, Math. SSSR Izv. 1 (1967), 391-420.

[27] M. S. Birman And M. Z. Solomjak, Spectral Theory of Self-Adjoint Operators in Hilbert Space, Reidel, Dordrecht, 1987.

[28] J. F. BRAsche, M. Malamud, AND H. NeIDHARDT, Weyl function and spectral properties of self-adjoint extensions, Integr. Eq. Oper. Th. 43 (2002), 264-289.

[29] M. S. BRods KII, Triangular and Jordan Representations of Linear Operators, Transl. Math. Mongraphs, Vol. 32, Amer. Math. Soc., Providence, RI, 1971.

[30] D. Buschmann, Spektraltheorie verallgemeinerter Differentialausdrücke - Ein neuer Zugang, Ph.D. Thesis, University of Frankfurt, Germany, 1997.

[31] R. W. CAREy, A unitary invariant for pairs of self-adjoint operators, J. Reine Angew. Math. 283 (1976), 294-312.

[32] J. CRUZ-SAMPEDRo, Exact asymptotic behavior at infinity of solutions to abstract second-order differential inequalities in Hilbert spaces, Math. Z. 237 (2001), 727-735. 
[33] Ju. L. Daleckii AND M. G. KREĬN, Stability of Solutions of Differential Equations in Banach Space, Transl. Math. Monographs, Vol. 43, Amer. Math. Soc., Providence, RI, 1974.

[34] L. DE BRAnges, Perturbations of self-adjoint transformations, Amer. J. Math. 84 (1962), 543-560.

[35] S. A. DEnisov, Schrödinger operators and associated hyperbolic pencils, J. Funct. Anal. 254 (2008), 2186-2226.

[36] V. A. DERKACH AND M. M. MaLAmud, Generalized resolvents and the boundary value problems for Hermitian operators with gaps, J. Funct. Anal. 95 (1991), 1-95.

[37] V. A. Derkach And M. M. Malamud, The extension theory of Hermitian operators and the moment problem, J. Math. Sci. 73 (1995), 141-242.

[38] V. A. DERKACH AND M. M. MaLAmud, On some classes of holomorphic operator functions with nonnegative imaginary part, in Operator Algebras and Related Topics, 16th International Conference on Operator Theory, A. Gheondea, R. N. Gologan, and T. Timotin (eds.), The Theta Foundation, Bucharest, 1997, pp. 113-147.

[39] J. Diestel And J. J. Uhl, Vector Measures, Mathematical Surveys, Vol. 15, Amer. Math. Soc., Providence, RI, 1977.

[40] J. Dieudonné, Foundations of Modern Analysis, Pure and Appl. Math., Vol. 10, Academic Press, New York, 1960.

[41] N. Dunford And J. T. Schwartz, Linear Operators Part II: Spectral Theory, Interscience, New York, 1988.

[42] D. E. Edmunds And W. D. Evans, Spectral Theory and Differential Operators, Clarendon Press, Oxford, 1989.

[43] P. Fillmore, Notes on Operator Theory, Math. Studies, Vol. 30, Van Nostrand-Reinhold, New York, 1970.

[44] F. Gesztesy, N.J. Kalton, K. A. Makarov, and E. Tsekanovskit, Some applications of operator-valued Herglotz functions, in "Operator Theory, System Theory and Related Topics," Oper. Theory Adv. Appl., Vol. 123, Birkhäuser, Basel, 2001, pp. 271-321.

[45] F. Gesztesy and E. Tsekanovskit, On matrix-valued Herglotz functions, Math. Nachr. 218 (2000), 61-138.

[46] M. L. GORBAČUK, On spectral functions of a second order differential operator with operator coefficients, Ukrain. Math. J. 18, No. 2, 3-21 (1966). (Russian.) Engl. transl. in Amer. Math. Soc. Transl. (2), 72 (1968), 177-202.

[47] V. I. GORBAČUK AND M. L. GORBAČUK, Expansion in eigenfunctions of a second-order differential equation with operator coefficients, Sov. Math. Dokl. 10 (1969), 158-162.

[48] M. L. GORBACHUK, Self-adjoint boundary problems for a second-order differential equation with unbounded operator coefficient, Funct. Anal. Appl. 5 (1971), 9-18.

[49] V. I. GorbACHUK AND M. L. GORBACHUK, The spectrum of self-adjoint extensions of the minimal operator generated by a Sturm-Liouville equation with operator potential, Ukrain. Math. J. 24 (1973), 582-588.

[50] V. I. Gorbachuk AND M. L. Gorbachuk, Boundary Value Problems for Operator Differential Equations, Kluwer, Dordrecht, 1991.

[51] M. L. GorbachuK AND V. A. MihaĬLEC, Semibounded selfadjoint extensions of symmetric operators, Sov. Math. Dokl. 17 (1976), 185-187.

[52] E. GüL, On the regularized trace of a second order differential operator, Appl. Math. Comp. 198 (2008), 471-480.

[53] E. Hille, Lectures on Ordinary Differential Equations, Addison-Wesley, Reading, MA, 1969.

[54] E. Hille And R. S. Phillips, Functional Analysis and Semi-Groups, Colloquium Publications, Vol. 31, rev. ed., Amer. Math. Soc., Providence, RI, 1985.

[55] D. Hinton AND A. SCHNEIDER, On the spectral representation for singular selfadjoint boundary eigenvalue problems, in Contributions to Operator Theory in Spaces with an Indefinite Metric, A. Dijksma, I. Gohberg, M. A. Kaashoek, R. Mennicken (eds.), Operator Theory: Advances and Applications, Vol. 106 (1998), pp. 217-251.

[56] W. J̈̈GER, Ein gewöhnlicher Differentialoperator zweiter Ordnung für Funktionen mit Werten in einem Hilbertraum, Math. Z. 113 (1970), 68-98.

[57] I. S. KAC AND M. G. KREIN, R-functions-analytic functions mapping the upper halfplane into itself, Amer. Math. Soc. Transl. (2) 103 (1974), 1-18. 
[58] T. KATO, Growth properties of solutions of the reduced wave equation with a variable coefficient, Commun. Pure Appl. Math. 12 (1959), 403-425.

[59] T. Kato, Perturbation Theory for Linear Operators, corr. printing of the 2nd ed., Springer, Berlin, 1980.

[60] A. G. Kostyuchenko And B. M. Levitan, Asymptotic behavior of the eigenvalues of the SturmLiouville operator problem, Funct. Anal. Appl. 1 (1967), 75-83.

[61] M. G. KREIN AND I. E. OvČARENKo, $Q$-functions and sc-resolvents of nondensely defined Hermitian contractions, Sib. Math. J. 18 (1977), 728-746.

[62] M. G. KREIN AND I. E. OvČARENKO, Inverse problems for $Q$-functions and resolvent matrices of positive Hermitian operators, Sov. Math. Dokl. 19 (1978), 1131-1134.

[63] A. Laptev And T. WeidL, Sharp Lieb-Thirring inequalities in high dimensions, Acta Math. 184 (2000), 87-111.

[64] A. LAPTEV, S. NABOKO, AND O. SAFRONOv, Absolutely continuous spectrum of Schrödinger operators with slowly decaying and oscillating potentials, Commun. Math. Phys. 253 (2005), 611631.

[65] J. S. Mac Nerney, Hermitian moment sequences, Trans. Amer. Math. Soc. 103 (1962), 45-81.

[66] M. M. Malamud And S. M. Malamud, On the spectral theory of operator measures, Funct. Anal. Appl. 36 (2002), 154-158.

[67] M. M. Malamud And S. M. Malamud, On the spectral theory of operator measures in Hilbert space, St. Petersburg Math. J. 15 (2004), 323-373.

[68] M. Malamud AND H. NeIDHARDT, On the unitary equivalence of absolutely continuous parts of self-adjoint extensions, J. Funct. Anal. 260 (2011), 613-638.

[69] M. Malamud And H. NeIdhardT, Sturm-Liouville boundary value problems with operator potentials and unitary equivalence, J. Diff. Eq. 252 (2012), 5875-5922.

[70] J. Mikusiński, The Bochner Integral, Academic Press, New York, 1978.

[71] P. A. MishnaEVSKII, On the spectral theory for the Sturm-Liouville equation with operator coefficient, Math. USSR Izv. 10 (1976), 145-180.

[72] P. A. MishnaevskiI, An investigation of a second-order elliptic operator in regions with infinite boundaries, by means of an operational Sturm-Liouville equation. I, Diff. Eq. 19 (1983), 334-344.

[73] P. A. MishnaevskiI, An investigation of a second-order elliptic operator in regions with infinite boundaries, by means of an operational Sturm-Liouville equation. II, Diff. Eq. 19 (1983), 475-483.

[74] V. I. MogILEVSKII, Description of spectral functions of differential operators with arbitrary deficiency indices, Math. Notes 81 (2007), 553-559.

[75] V. MogiLeVs KII, Boundary triplets and Titchmarsh-Weyl functions of differential operators with arbitrary deficiency indices, Meth. Funct. Anal. Topology 15 (2009), 280-300.

[76] V. MogILEVSKII, Minimal spectral functions of an ordinary differential operator, arXiv:1010.1117.

[77] S. N. NАBOKO, Boundary values of analytic operator functions with a positive imaginary part, J. Soviet Math. 44 (1989), 786-795.

[78] S. N. NAвоKо, Nontangential boundary values of operator-valued $R$-functions in a half-plane, Leningrad Math. J. 1 (1990), 1255-1278.

[79] S. N. NABOKO, The boundary behavior of $\mathfrak{S}_{p}$-valued functions analytic in the half-plane with nonnegative imaginary part, Functional Analysis and Operator Theory, Banach Center Publications, Vol. 30, Institute of Mathematics, Polish Academy of Sciences, Warsaw, 1994, pp. 277-285.

[80] M. A. Naimark, Linear Differential Operators, Part II, Ungar, New York, 1968.

[81] F. J. NARCOWICH, Mathematical theory of the $R$ matrix. II. The $R$ matrix and its properties, J. Math. Phys. 15 (1974), 1635-1642.

[82] F. J. NARCOWICH, $R$-operators II. On the approximation of certain operator-valued analytic functions and the Hermitian moment problem, Indiana Univ. Math. J. 26 (1977), 483-513.

[83] F. J. Narcowich And G. D. Allen, Convergence of the diagonal operator-valued Padé approximants to the Dyson expansion, Commun. Math. Phys. 45 (1975), 153-157.

[84] B. J. PetTis, On integration in vector spaces, Trans. Am. Math. Soc. 44 (1938), 277-304.

[85] A. I. Plesner AND V. A. Rohlin, Spectral theory of linear operators, Uspehi Matem. Nauk (N. S.) 1(11), No. 1, 71-191 (1946). (Russian.) Engl. transl. in Amer. Math. Soc. Transl. (2), 62 (1967), 29-175.

[86] F. RiesZ AND B. SZ.-NAGY, Functional Analysis, Dover, New York, 1990. 
[87] F. S. Rofe-BEKETOV, Expansions in eigenfunctions of infinite systems of differential equations in the non-self-adjoint and self-adjoint cases, Mat. Sb. 51 (1960), 293-342. (Russian.)

[88] F. S. RoFE-BEKETOV, Selfadjoint extensions of differential operators in a space of vector functions, Sov. Math. Dokl. 10 (1969), 188-192.

[89] F. S. Rofe-BEKETOv, Selfadjoint extensions of differential operators in a space of vector-valued functions, Teor. Funkcii Funkcional. Anal. i Priložen. Vyp. 8 (1969), 3-24. (Russian.)

[90] F. S. RofE-BEKeTOV AND A. M. KHOLKIN, A fundamental system of solutions of for an operator differential equation with a boundary condition at infinity, Math. Notes 36 (1985), 846-853.

[91] F. S. Rofe-Beketov And A. M. Kholkin, Spectral Analysis of Differential Operators. Interplay Between Spectral and Oscillatory Properties, Monograph Series in Mathematics, Vol. 7, World Scientific, Singapore, 2005.

[92] O. SAFRONOV, Absolutely continuous spectrum of one random elliptic operator, J. Funct. Anal. 255 (2008), 755-767.

[93] O. SAFronov AND G. STOLZ, Absolutely continuous spectrum of Schrödinger operators with potentials slowly decaying inside a cone, J. Math. Anal. Appl. 326 (2007), 192-208.

[94] Y. SAITO, Eigenfunction expansions associated with second-order differential equations for Hilbert space-valued functions, Publ. RIMS, Kyoto Univ. 7 (1971/72), 1-55.

[95] Y. SAITO, The principle of limiting absorption for second-order differential equations with operatorvalued coefficients, Publ. RIMS, Kyoto Univ. 7 (1971/72), 581-619.

[96] Y. SAITO, Spectral and scattering theory for second-order differential operators with operatorvalued coefficients, Osaka J. Math. 9 (1972), 463-498.

[97] Y. SAITO, Spectral theory for second-order differential operators with long-range operator-valued coefficients II. Eigenfunction expansions and the Schrödinger operators with long-range potentials, Japan. J. Math. 1 (1975), 311-349.

[98] Y. SAITO, Spectral theory forsecond-order differential operators with long-range operator-valued coefficients I. Limiting absorption principle, Japan. J. Math. 1 (1975), 351-382.

[99] Y. SAITO, On the asymptotic behavior of solutions of the Schrödinger equation $\left(-\Delta+Q(y)-k^{2}\right) V=$ $F$, Osaka J. Math. 14 (1977), 11-35.

[100] Y. SAITO, Eigenfunction expansions for the Schrödinger operators with long-range ptentials $\left(Q(y)=O\left(|y|^{-\varepsilon}\right), \varepsilon>0\right.$, Osaka J. Math. 14 (1977), 37-53.

[101] Y. S AITO, Spectral Representations for Schrödinger Operators with Long-Range Potentials, Lecture Notes in Mathematics, Vol. 727, Springer, Berlin, 1979.

[102] Yu. L. Shmul'yan, On operator R-functions, Siberian Math. J. 12 (1971), 315-322.

[103] I. Trooshin, Asymptotics for the spectral and Weyl functions of the operator-valued SturmLiouville problem, in Inverse Problems and Related Topics, G. Nakamura, S. Saitoh, J. K. Seo, and M. Yamamoto (eds.), Chapman \& Hall/CRC, Boca Raton, FL, 2000, pp. 189-208.

[104] E. R. TSEKANOVSKII, Accretive extensions and problems on the Stieltjes operator-valued functions realizations, in Operator Theory and Complex Analysis, T. Ando and I. Gohberg (eds.), Operator Theory: Advances and Applications, Vol. 59, Birkhäuser, Basel, 1992, pp. 328-347.

[105] L. I. VAinerman AND M. L. GorbaChUK, On self-adjoint semibounded abstract differential operators, Ukrain. Math. J. 22 (1970), 694-696.

[106] J. Weidmann, Linear Operators in Hilbert Spaces, Graduate Texts in Mathematics, Vol. 68, Springer, New York, 1980.

[107] J. Weidmann, Spectral Theory of Ordinary Differential Operators, Lecture Notes in Math. 1258, Springer, Berlin, 1987.

[108] H. WEYL, "Uber gewöhnliche Differentialgleichungen mit Singularitäten und die zugehörigen Entwicklungen willkürlicher Funktionen, Math. Ann. 68 (1910), 220-269.

[109] K. Yosida, Functional Analysis, 6th ed., Springer, Berlin, 1980. 